Article

\title{
Prophylactic Corneal Cross-Linking in Myopic Femtosecond Laser-Assisted In Situ Keratomileusis: Long-Term Visual and Refractive Outcomes
}

\author{
Federico Alonso-Aliste ${ }^{1}$, Jonatan Amián-Cordero ${ }^{1}$, Rahul Rachwani-Anil ${ }^{2}{ }^{\mathbb{D}}$, Concepción De-Hita-Cantalejo ${ }^{3}$, \\ Davide Borroni $^{4,5}$ (D), Carlos Rocha-de-Lossada ${ }^{6,7,8}$ (D) and José-María Sánchez-González ${ }^{3, *(D)}$
}

1 Department of Ophthalmology, Tecnolaser Clinic Vision, 41018 Seville, Spain; federicornea@gmail.com (F.A.-A.); dramian@tecnolasersevilla.com (J.A.-C.)

2 Department of Ophthalmology, Hospital Regional de Málaga, 29010 Málaga, Spain; rahul.medum@gmail.com

3 Department of Physics of Condensed Matter, Optics Area, University of Seville, 41012 Seville, Spain; mhita@us.es

4 Fondazione Banca Degli Occhi Del Veneto Onlus, Zelarino, 30174 Venezia, Italy; info.borroni@gmail.com

5 Department of Doctoral Studies, Riga Stradins University, LV-1007 Riga, Latvia

6 Department of Ophthalmology (Qvision), Vithas Virgen del Mar Hospital, 04120 Almería, Spain; carlosrochadelossada5@gmail.com

7 Department of Ophthalmology, Hospital Virgen de las Nieves, 18014 Granada, Spain

8 Department of Ophthalmology, Ceuta Medical Center, 51001 Ceuta, Spain

* Correspondence: jsanchez80@us.es

check for updates

Citation: Alonso-Aliste, F; Amián-Cordero, J.; Rachwani-Anil, R.; De-Hita-Cantalejo, C.; Borroni, D.;

Rocha-de-Lossada, C.;

Sánchez-González, J.-M. Prophylactic Corneal Cross-Linking in Myopic Femtosecond Laser-Assisted In Situ Keratomileusis: Long-Term Visual and Refractive Outcomes. Optics 2021, 2, 55-62. https://doi.org/10.3390/ opt2010006

Received: 18 February 2021

Accepted: 10 March 2021

Published: 15 March 2021

Publisher's Note: MDPI stays neutral with regard to jurisdictional claims in published maps and institutional affiliations.

Copyright: (c) 2021 by the authors. Licensee MDPI, Basel, Switzerland. This article is an open access article distributed under the terms and conditions of the Creative Commons Attribution (CC BY) license (https:/ / creativecommons.org/licenses/by/ $4.0 /)$.

\begin{abstract}
The purpose of our study was to evaluate the safety, effectiveness, predictability, and stability of myopic and astigmatic laser-assisted in situ keratomileusis (LASIK) with simultaneous prophylactic corneal cross-linking (CXL) in thin corneas. In total, 100 eyes from 50 patients who were subjected to myopic and astigmatism femtosecond LASIK with simultaneous prophylactic CXL were included. The design of the study was retrospective, longitudinal, and observational. All patients had a 48-month follow-up. The MEL 80 excimer laser was utilized with the Aberration Smart Ablation platform. CXL treatment was applied when the predicted stromal thickness was less than $330 \mu \mathrm{m}$. Patients' mean age was $30.22 \pm 5.97$ years. Previous mean spherical equivalent was $-5.50 \pm 1.65$ ( -9.50 to -1.13$)$ diopters (D). Postoperative mean spherical equivalent was $-0.24 \pm 0.29(-0.85$ to +0.50 ) D. Visual acuity (VA) of $20 / 20$ or better was observed in $87 \%$ of the eyes and no eyes experienced VA loss. Spherical equivalent within $\pm 0.50 \mathrm{D}$ was observed in $93 \%$ of eyes, and $4 \%$ of eyes varied by $0.50 \mathrm{D}$ or more between 3 and 48 months. Prophylactic corneal cross-linking with simultaneous femtosecond laser-assisted in situ keratomileusis in thin corneas proved to be effective, safe, and predictable. The results remained stable after 48 months of follow-up.
\end{abstract}

Keywords: cross-linking; LASIK; femtosecond; FemtoLASIK

\section{Introduction}

Laser-assisted in situ keratomileusis (LASIK) is the most common and well-known refractive surgery procedure [1]. LASIK is safe and effective in treating myopia, astigmatism, and both in combination. However, iatrogenic ectasia and refractive regression remain some of the main research concerns [2]. Post-LASIK ectasia (PLE) is an unusual although serious complication that could take place early on or up to several years following the refractive surgery procedure. Furthermore, it is ordinary following high myopic correction, even though it can happen following hyperopic treatment [3]. LASIK requires a corneal flap creation using a femtosecond laser or a microkeratome and it weakens the cornea structure as well as decreases corneal biomechanics and rigidity [4]. Refractive regression and iatrogenic ectasia are well-known complications as a consequence of structural changes after LASIK. PLE risk factors comprise high myopia or hyperopia, thin central corneal 
thickness, low residual stromal bed after surgery, and subjects with atypical topography (such as fruste keratoconus) [5]. PLE implies loss of uncorrected distance visual acuity (UDVA) and, in many cases, loss of corrected distance visual acuity (CDVA) as a result of corneal steepening that appears centrally or inferiorly. The estimated reported incidence of PLE ranges from $0.04 \%$ to $0.60 \%$ [5].

Prophylactic corneal cross-linking (CXL) has emerged in order to prevent PLE [6] in eyes with low residual stromal bed or in thin corneas. Corneal biomechanical behavior could be altered by a low concentration of riboflavin and ultraviolet (UV) radiation [7]. CXL consists in stromal fiber photopolymerization by the combined action of riboflavin (photosensitizing substance) and ultraviolet-A (UVA) light [8]. Thus, corneal stroma gains tensile strength and fiber stability [2]. Wollensak et al. [9] demonstrated that the biomechanical effect of CXL using the femtosecond laser pocket technique is about $50 \%$ less aggressive than that after standard CXL. Kampik et al. [10] reported that CXL reduces the amount of refractive change after LASIK for myopia, although the laser ablation rate is unaffected. Previous authors have already studied prophylactic CXL and LASIK with short or medium follow-up [8,11-19]. To the best of our knowledge, this is the longest follow-up study regarding prophylactic CXL with simultaneous LASIK.

The purpose of our study was to evaluate the safety, effectiveness, predictability, and endurance of myopic and astigmatic LASIK with simultaneous prophylactic corneal CXL.

\section{Materials and Methods}

\subsection{Design}

In total, 100 eyes from 50 patients who were subjected to myopic and astigmatism femtosecond laser-assisted in situ keratomileusis (LASIK) with simultaneous prophylactic CXL were included in this retrospective, observational, longitudinal study. Patients underwent surgery between January 2014 and December 2015. All surgeries were performed at the facilities of the Ophthalmology Center Tecnolaser Clinic Vision ${ }^{\circledR}$, Seville, Spain. All patients had a 48-month follow-up.

\subsection{Ethical Aspects}

All patients included in this work were adequately informed, verbally and in writing, of the benefits, characteristics, and risks of the surgeries. All patients signed an informed consent form prior to the surgery and after the interview with the ophthalmologist. This study was conducted in accordance with the tenets of the Helsinki Declaration and received approval by the institution's ethics committee.

\subsection{Subjects}

Fifty patients ( 28 women and 22 men) willingly presented to the clinic to undergo the clinical examinations. After being assessed by the ophthalmologist and meeting the requirements for surgery, they were scheduled for myopic and astigmatism femtosecond LASIK surgery. The inclusion criteria were (1) bilateral myopia or myopia with astigmatism, (2) age older than 18 years and less than 45 years, (3) an unchanged refraction in the past year-that is, a change of $\leq 0.50$ diopters (D) in the spherical and cylindrical refraction-(4) myopia in spherical equivalent (MSRE) between -1.00 and $-10.00 \mathrm{D}$, (5) astigmatism between 0.00 and $-5.00 \mathrm{D}$, (6) best preoperative corrected distance visual acuity of 20/30 or better in each eye, (7) calculated residual stromal bed of $330 \mu \mathrm{m}$ or less, (8) less than $10 \mathrm{D}$ difference between the maximum and minimum corneal curvature values, and (9) $\leq 0.50-\mathrm{D}$ difference between two keratometric values measured with at least a one-week gap in contact lens wearers. Contact lens wearers were advised not to use them at least 15 days before surgery. The exclusion criteria were (1) corneal diseases, (2) ocular surface diseases, (3) retinal disorders, (4) immunodeficiency or connective tissue disorders, (5) pregnancy or breastfeeding, (6) intolerance or allergy to any of the medications in the postoperative treatment for LASIK, (7) eye muscle diseases or lack of eye fixation, and (8) blindness or amblyopia in the fellow eye. 


\subsection{Preoperative Examinations}

Before undergoing femtosecond LASIK with prophylactic CXL surgery, a thorough preoperative examination was carried out in all patients. The examination was carried out by an experienced optometrist and it consisted of distance uncorrected and corrected visual acuity (decimal and Snellen ratio), subjective non-cycloplegic refraction, and cycloplegic refraction by the fogging technique. Astigmatism was measured using the Jackson crosscylinder method. Data were validated with a Wavefront Aberration Supported Custom Ablation (WASCA) aberrometer and autorefractor (Carl Zeiss Meditec AG, Jena, Germany). Strabismus and convergence were studied in all patients. A Pentacam ${ }^{\circledR}$ single rotation Scheimpflug camera (Oculus Optikgeräte $\mathrm{GmbH}$, Wetzlar, Germany) was employed to carry out corneal topography as well as keratometry and pachymetry. A Perkins Mk3 hand-held applanation tonometer was used to assess intraocular pressure (Haag-Streit, UK). Corneal epithelium and retinal examination were assessed using spectral domain optical coherence tomography (SD-OCT) (Optovue Inc., Fremont, CA, USA). Finally, refraction was verified once again by a different optometrist from the one who had performed the first examination, before performing the surgery.

\subsection{Surgical Technique}

All the surgical interventions were carried out by two experienced ophthalmologists in FemtoLASIK correction (F.A.A and J.A.C). Povidone-iodine 5\% (Betadine; Meda Manufacturing, Bordeaux, France) was applied on the eye contour for disinfection $10 \mathrm{~min}$ prior to surgery. Subsequently, both eyes were instilled with a drop of double anesthetic (tetracaine $0.1 \%$ and oxybuprocaine $0.4 \%$ ) (Alcon Cusí, El Masnou, Barcelona, Spain).

The patient was placed on the table under the cone. Later, the flap was performed using the VisuMax Femtosecond Laser System (Carl Zeiss Meditec AG, Jena, Germany). The laser was centered on the patient's pupil. We requested the patient to stare at the green light inside the cone. The pulse energy was established at approximately $130 \mathrm{~nJ}$. The laser frequency was set at $500 \mathrm{KHz}$. The spot distance was established at $4.5 \mu \mathrm{m}$. The estimated flap thickness was $100 \mu \mathrm{m}$. The flap diameter was $8.5 \mathrm{~mm}$.

Excimer laser treatment was performed with the MEL 80 excimer laser system (Carl Zeiss Meditec AG, Jena, Germany). The laser type was excimer pulsed argon and fluoride (ArF). The wavelength was $193 \mathrm{~nm}$. The beam dimension was $0.7 \mathrm{~mm}$ full width at half maximum (FWHM) with a Gaussian beam profile. The frequency was $250 \mathrm{~Hz}$. The treatment was centered in coaxially sighted corneal light reflex. An Aberration Smart Ablation (ASA) profile was used for all myopic and astigmatism corrections into a single profile to decrease ablation depth. The target was plano and the optical zone was set from 6.00 to $6.50 \mathrm{~mm}$.

\subsubsection{Corneal Cross-Linking}

After excimer ablation with the flap folded, one drop of Vibex Rapid TM (Avedro, Inc., Waltham, MA, USA) containing $0.25 \%$ saline-diluted riboflavin mixed with balanced salt solution was placed on the stromal bed. The corneal stromal bed was saturated with the solution for $90 \mathrm{~s}$. Excess of riboflavin was washed out, and the flap was then placed into its initial position. The surface was then irradiated with $30 \mathrm{~mW} / \mathrm{cm}^{2}$ ultraviolet light of $375 \mathrm{~nm}$ using the KXL System ${ }^{\circledR}$ (Avedro, MA, USA) for $90 \mathrm{~s}$ with a total energy of $2.7 \mathrm{~J} / \mathrm{cm}^{2}$ and a diameter of the treatment area of $9.00 \mathrm{~mm}$. Corneal cross-linking data are reported in Table 1, according to the standard terminology and protocol nomenclature [20]. To avoid risk of accidental flap contact, a neutral power bandage contact lens (Comfilcon A, Biofinity with a $14.00-\mathrm{mm}$ diameter and a $8.6-\mathrm{mm}$ radius, CooperVision, Lake Forest, CA, USA) was placed at the end of surgery and removed the following day.

\subsubsection{Postoperative Evaluation}

Patients were trained to use soft eye patches before sleeping for two nights. Tobramycin $0.3 \%$, dexamethasone $0.1 \%$, and fluorometholone $0.3 \%$ were applied five times 
daily for the first week and then three times daily for the second week. Treatment was then tapered until complete withdrawal. Patients were revised at day one, 15 days, and 3, 12, and 48 months after surgery.

Table 1. Prophylactic cross-linking parameters.

\begin{tabular}{cc}
\hline Parameter & Variable \\
\hline Treatment target & Prophylaxis \\
Fluence (total) $\left(\mathrm{J} / \mathrm{cm}^{2}\right)$ & 2.7 \\
Soak time $(\mathrm{s})$ & 90 \\
Intensity $(\mathrm{mW})$ & 30 \\
Treatment time (seconds) & 90 \\
Epithelium status & On (Flap folded) \\
Chromophore & Riboflavin (Vibex Rapid Avedro) \\
Chromophore carrier & Balanced Salt Solution \\
Chromophore osmolarity & Iso-Osmolar \\
Chromophore concentration & $0.25 \%$ \\
Light source & UVA (KXL System, Avedro) \\
Irradiation mode (interval) & Continuous \\
Protocol modifications & None \\
Protocol abbreviation in manuscript & Prophylactic CXL \\
\hline
\end{tabular}

\subsection{Statistical Analysis}

Statistical analysis was carried out with SPSS statistics 26.0 (IBM Corporation, Armonk, NY, USA). All visual acuity data were converted into Snellen format. The Student t-test was performed for parametric dependent variables. All statistical tests were performed with a $95 \%$ confidence level $(p<0.05)$.

\section{Results}

Patients' mean age was $30.22 \pm 5.97$ (22 to 44) years. Prior to surgery, the mean sphere was $-4.92 \pm 1.58(-8.25$ to -1.00$) \mathrm{D}$, the mean cylinder was $-1.15 \pm 1.17(-4.50$ to 0.00$) \mathrm{D}$, and the mean spherical equivalent was $-5.50 \pm 1.65(-9.50$ to -1.13$) \mathrm{D}$. The postoperative mean sphere at 48 months follow-up was $-0.09 \pm 0.30(-0.75$ to +1.00$) \mathrm{D}$, the mean cylinder was $-0.29 \pm 0.33(-1.25$ to +0.50$) \mathrm{D}$, and the mean spherical equivalent was $-0.24 \pm 0.29(-0.85$ to +0.50$)$ D. Preoperative uncorrected distance visual acuity (UDVA) was $20 / 196.60 \pm 19.70(20 / 200$ to 20/60). Pre-surgical corrected distance visual acuity (CDVA) was 20/21.20 \pm 2.37 (20/32 to 20/20). Postoperative UDVA at 48 months follow-up was $20 / 20.20 \pm 2.74(20 / 32$ to $20 / 16)$.

Efficacy in terms of distance cumulative Snellen visual acuity (20/x or better) is presented in Figure 1A. In total, $98 \%$ of the eyes obtained $20 / 25$ or better, $87 \%$ of the eyes obtained $20 / 20$ or better, and $11 \%$ of the eyes obtained $20 / 16$ or better. Efficacy index as a result of postoperative UDVA divided by CDVA was 0.95 . Regarding safety, no eyes lost one or more lines and the results are presented in Figure 1B. The achieved spherical equivalent refraction versus the targeted spherical equivalent refraction is presented in Figure 1C. Postoperative spherical equivalent refraction accuracy data are presented in Figure 1D. Postoperative refractive astigmatism data are presented in Figure 1E. Finally, regarding stability, the preoperative spherical equivalent was $-5.50 \pm 1.65 \mathrm{D}$, and 48 months later, it changed to $-0.24 \pm 0.29 \mathrm{D}$, out of which $4 \%$ of eyes suffered a $0.50-\mathrm{D}$ variation or more between 3 and 48 months (Figure 1F). At the third month of follow-up, two patients did not attend their appointment, and at month 24, five patients did not attend their appointment. Regarding reported complications, two eyes needed an enhancement for distance. Retreatment was performed under the same excimer technique without corneal reinforcement. Two eyes with striae requiring flap re-lift and irrigation were identified. One eye with adenovirus conjunctivitis was reported. No eyes had PLE after surgery. 


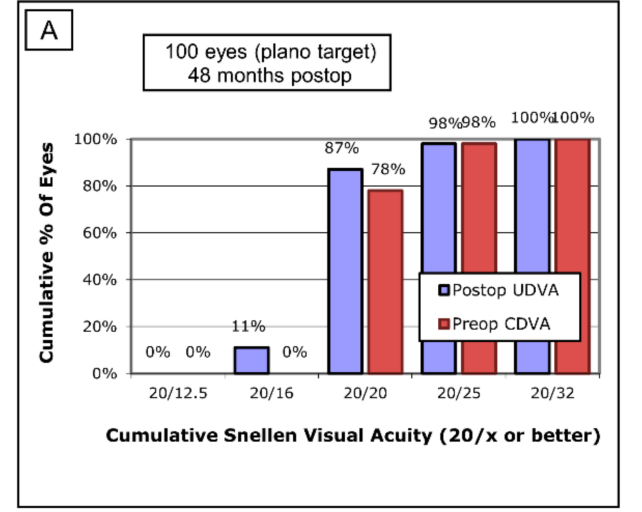

Uncorrected Distance Visual Acuity

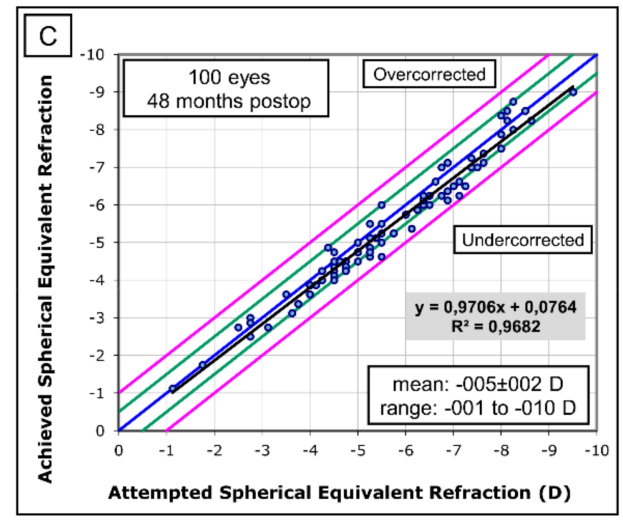

Spherical Equivalent Attempted vs Achieved

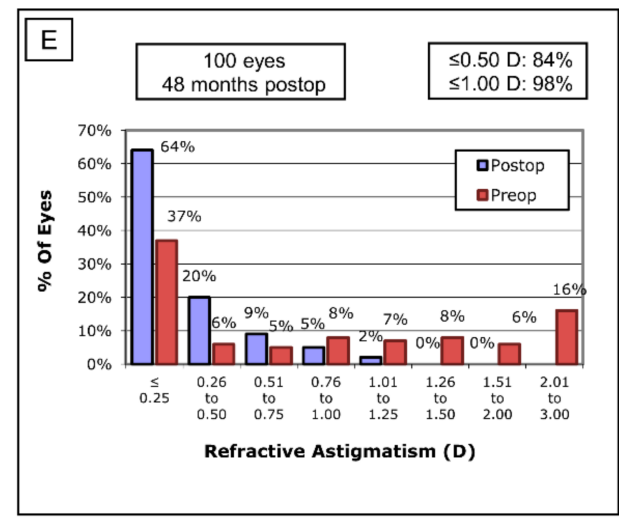

Refractive Astigmatism

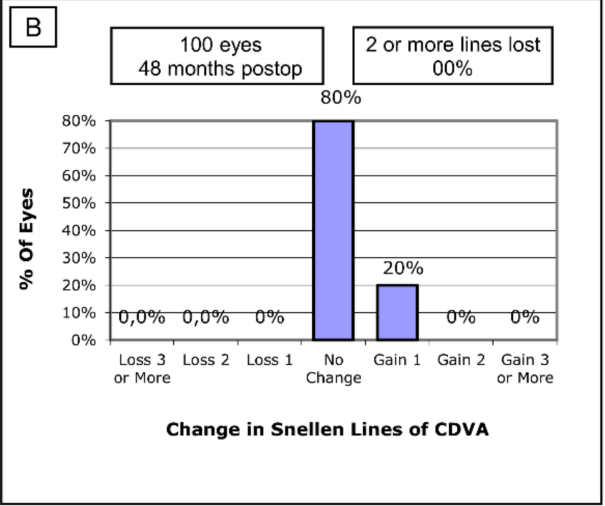

Change in Corrected Distance Visual Acuity

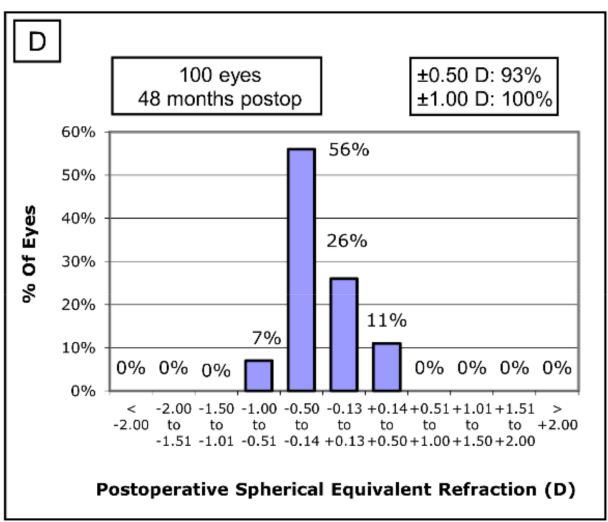

Spherical Equivalent Refractive Accuracy

Figure 1. Femtosecond laser-assisted in situ keratomileusis (LASIK) with simultaneous prophylactic corneal cross-linking (CXL) standard graphs for reporting refractive surgery. (A) Uncorrected distance visual acuity (UDVA) —efficacy histogram. (B) Change in corrected distance visual acuity (CDVA) safety histogram. (C) Spherical equivalent attempted versus achieved. (D) Spherical equivalent refractive accuracy. (E) Refractive astigmatism. Graphs (C-E) represent predictability. (F) Stability of spherical equivalent refraction.

\section{Discussion}

Our retrospective study reported visual and refractive outcomes after performing prophylactic CXL with simultaneous femtosecond LASIK surgery in 100 myopic and astigmatic eyes after 48 months of follow-up. We reported efficacy, safety, predictability, and stability. To the best of our knowledge, this publication has the biggest sample size and the longest follow-up of its kind. 
Regarding effectiveness, we observed that $87 \%$ accomplished $20 / 20$, and all patients improved their UDVA (Figure 1A). Other studies [8,11-19] similar to our work are reported in Table 2. Some authors $[11-13,15,16,19]$ found similar results to ours, while others $[8,17,18]$ reported worse efficacy results in UDVA. However, studies with unfavorable results share small sample sizes or short follow-up periods. Comparative studies reported similar or better long-term visual and refractive postoperative spherical equivalent, efficacy index, UDVA, or refraction. The efficacy index in prophylactic CXL with femtosecond LASIK in myopia ranges from 0.99 to 1.16 [14-16,19]. In a comparative study in high myopia patients, Kanellopoulus et al. [12] reported that prophylactic CXL LASIK and LASIK only had a similar spherical equivalent refraction, with $84.6 \%$ and $81.3 \%$ eyes within $\pm 0.50 \mathrm{D}$, respectively.

Table 2. Prophylactic CXL with LASIK results among previous studies. Efficacy. Percentage postoperative uncorrected binocular distance and visual acuity (UDVA) (with 20/20 or better) (Efficacy index is also shown if available). Safety. Percentage of eyes with corrected distance visual acuity (CDVA) with one and two lines of loss or more. Predictability. Percentage of eyes with spherical equivalent refraction accuracy within \pm 0.50 and $\pm 1.00 \mathrm{D}$. Stability. Percentage of eyes with a change of $0.50 \mathrm{D}$ or more between an intermediate and final month of follow-up. Follow-up is reported in months. LASIK procedure is reported first flap technique and second excimer laser platform.

\begin{tabular}{|c|c|c|c|c|c|c|c|c|}
\hline Author & Year & Corneal CXL & LASIK & Eyes & Follow-up & Efficacy & Safety & Predictability \\
\hline Celik et al. [8] & 2012 & Avedro & MK and Schwind & 4 & 12 & $75 \% / 1.06$ & $0 \% / 0 \%$ & $100 \% / 100 \%$ \\
\hline Kanellopoulus [16] & 2012 & Priavision & FS and Wavelight & 43 & 42 & $83.7 \% / 1.09$ & $0 \% / 0 \%$ & - \\
\hline Tomita et al. [15] & 2014 & Avedro & FS and Schwind & 24 & 12 & $100 \% / 0.99$ & $21.7 \% / 0 \%$ & $95.7 \% / 95.7 \%$ \\
\hline Kanellopoulus et al. [13] & 2014 & Avedro & FS and Wavelight & 73 & 12 & $90.4 \% /-$ & $0 \% / 0 \%$ & $91.8 \% / 100 \%$ \\
\hline Seiler et al. [14] & 2015 & UVX 2000 & FS and Wavelight & 76 & 12 & NR & $0 \% / 0 \%$ & $\approx 85 \% / \approx 95 \%$ \\
\hline Kanellopoulus et al. [12] & 2015 & Avedro & FS and Wavelight & 65 & 24 & $93.8 \% /-$ & $0 \% / 0 \%$ & $92.3 \% / 100 \%$ \\
\hline Tan et al. [17] & 2015 & Avedro & FS and Schwind & 70 & 3 & $78 \% /-$ & $17 \% / 0 \%$ & $100 \% / 100 \%$ \\
\hline Chang et al. [11] & 2016 & Avedro & FS and Allegretto & 60 & 6 & $86.7 \% / 0.91$ & $\approx 5 \% / 0 \%$ & $96 \% / 98 \%$ \\
\hline Wu et al. [19] & 2016 & Avedro & FS and Wavelight & 96 & 6 & $87.5 \% / 1.02$ & $2 \% / 2 \%$ & $-/ 95.83 \%$ \\
\hline Kohnen et al. [18] & 2020 & Avedro & FS and Schwind & 26 & 12 & $78 \% /-$ & $17 \% / 0 \%$ & $87 \% / 91 \%$ \\
\hline
\end{tabular}

CXL: Cross-linking; LASIK: laser-assisted in situ keratomileusis; MK: microkeratome; FS: femtosecond; NR: not reported.

In terms of safety, our results showed no eyes with one or more lines of loss in CDVA (Figure 1B). Other studies' safety results are presented in Table 2. Our results matched with some previously reported by other authors $[8,11-14,16,19]$ but disagree with the ones reported by Tomita et al. [15], Tan et al. [17], and Kohnen et al. [18]. In most cases, the safety matches or excels the results of LASIK alone. Tomita et al. [15] reported the highest loss in UDVA, although they did not find serious intraoperative complications in any eyes. Superficial punctate keratitis and corneal edema observed in slit-lamp examination appeared in two eyes. The complications observed in our study were independent from prophylactic CXL and could have appeared in a femtosecond LASIK surgery without prophylactic CXL. Celik et al. [8] reported the presence of faint stromal haze (0.5 grade) in CXL-LASIK in the first postoperative week of follow-up. Seiler et al. [14] described the prevalence of transient side effects such as diffuse lamellar keratitis (DLK) and delayed visual recovery. $\mathrm{Ng}$ et al. [21] had previously shown that the demarcation line achieved in prophylactic CXL with LASIK is similarly safe for 120 or $180 \mathrm{~s}$ of treatment. In their study, the intensity was $18 \mathrm{~mW}$, while in ours, it was $30 \mathrm{~mW}$ and the treatment time was only $90 \mathrm{~s}$. No study, including ours, has reported ectasia in prophylactic CXL and LASIK. However, Taneri et al. [22] described a young patient that developed unilateral corneal ectasia after undergoing a combined surgery of LASIK and prophylactic CXL, and with a normal presurgical ophthalmic examination. Consequently, from the currently available literature, only one case out of 638 eyes treated with prophylactic CXL and LASIK developed an iatrogenic ectasia. Although the aim of our study was not to compare CXL-LASIK versus other procedures that may be indicated in low residual stromal bed (RSB) eyes, such as phakic intraocular lens (IOL) or surface ablation, the reported literature stated above along with our results prove that LASIK-CXL is a safe option in patients with risk of PLE. 
In terms of predictability, our results obtained $0.9706 x+0.0764\left(R^{2}=0.9682\right)$. Most of the authors who reported the results of prophylactic CXL and LASIK did not present the predictability in terms of a regression line between the attempted refraction versus achieved refraction. Among the authors who have reported predictability data, there is a difference of opinions. Kanellopoulus et al. [13,23] and Kohnen et al. [18] obtained better results in prophylactic CXL-LASIK (1.011x + 0.011, 1.008x + 0.0416 (from Kanellopoulus) and 1.0406x +0.4501 (from Kohnen)). Tan et al. [17] and Chang et al. [11] obtained similar results with and without CXL and LASIK $(0.9752 x+0.1405$ and $1.0031 x+0.9539$, respectively). Finally, Tomita et al. [15] and Wu et al. [19] found better predictability results without simultaneous prophylactic CXL on LASIK (1.2129x -0.8565 and $0.8465 x-0.9539$, respectively). Regarding spherical equivalent, our results showed $93 \%$ eyes within $\pm 0.50 \mathrm{D}$ and all eyes within $\pm 1.00 \mathrm{D}$. Most of the previous studies $[8,11-13,15,17]$ showed similar spherical equivalent predictability, while others $[14,18]$ reported worse spherical equivalent predictability. All details are presented in Table 2.

In terms of stability, our outcomes proved a variation of $0.31 \mathrm{D}$ from the third month of follow-up $(+0.07 \mathrm{D})$ until the forty-eighth month $(-0.24 \mathrm{D})$. If we analyze the stability in the rest of the literature, studied with a follow-up equal to or greater than one year and with a significant sample size equal to or greater than 40 eyes, we can only identify three studies carried out by Kanellopoulus et al. [12,13,16] and one by Seiler et al. [14]. The first study of Kanellopoulus et al. [16] shows regression in terms of keratometry values, reported from month three $(37.53 \mathrm{D})$ to month $24(38.09 \mathrm{D})$ and expressed in mean keratometry. The second work by Kanellopoulus et al. [13] also reported keratometry changes at month three $(37.98 \mathrm{D})$ and after one year $(38.34 \mathrm{D})$, expressed in mean keratometry. The third study of Kanellopoulus et al. [12] reported similar findings. Finally, Seiler et al. [14] only reported one-year refractive differences between CXL-LASIK with $-0.05 \pm 0.41 \mathrm{D}$ and LASIK only with $-0.21 \pm 0.45 \mathrm{D}$. Despite the fact that there are very limited studies to compare with, all authors proved the presence of a slight regression. The limitations of our study are mainly that the design is retrospective and the lack of a control arm. A longer follow-up period is necessary to support the safety and predictability of simultaneous CXL and LASIK. Future studies comparing the outcomes of enhancement in CXL-LASIK vs. LASIK could be beneficial.

\section{Conclusions}

Prophylactic corneal cross-linking with simultaneous femtosecond laser-assisted in situ keratomileusis (LASIK) in our study proved to be effective, safe, predictable, and stable after 48 months of follow-up. Our results showed a minor refractive regression and excellent efficacy and safety results. A larger sample size and a longer follow-up are crucial to confirm the reported outcomes.

Author Contributions: Conceptualization, F.A.-A., J.A.-C., R.R.-A., C.D.-H.-C., D.B., C.R.-d.-L., J.-M.S.-G.; methodology, F.A.-A., J.A.-C., R.R.-A., C.D.-H.-C., D.B., C.R.-d.-L., J.-M.S.-G.; software, F.A.-A., J.A.-C., R.R.-A., C.D.-H.-C., D.B., C.R.-d.-L., J.-M.S.-G.; validation, F.A.-A., J.A.-C., R.R.-A., C.D.-H.-C., D.B., C.R.-d.-L., J.-M.S.-G.; formal analysis, F.A.-A., J.A.-C., R.R.-A., C.D.-H.-C., D.B., C.R.-d.-L., J.-M.S.-G.; investigation, F.A.-A., J.A.-C., R.R.-A., C.D.-H.-C., D.B., C.R.-d.-L., J.-M.S.-G.; resources, F.A.-A., J.A.-C., R.R.-A., C.D.-H.-C., D.B., C.R.-d.-L., J.-M.S.-G.; data curation, F.A.-A., J.A.-C., R.R.-A., C.D.-H.-C., D.B., C.R.-d.-L., J.-M.S.-G.; writing-original draft preparation, F.A.-A., J.A.-C., R.R.-A., C.D.-H.-C., D.B., C.R.-d.-L., J.-M.S.-G.; writing-review and editing, F.A.-A., J.A.-C., R.R.-A., C.D.-H.-C., D.B., C.R.-d.-L., J.-M.S.-G.; visualization, F.A.-A., J.A.-C., R.R.-A., C.D.-H.-C., D.B., C.R.-d.-L., J.-M.S.-G.; supervision, F.A.-A., J.A.-C., R.R.-A., C.D.-H.-C., D.B., C.R.-d.-L., J.-M.S.-G.; project administration, F.A.-A., J.A.-C., R.R.-A., C.D.-H.-C., D.B., C.R.-d.-L., J.-M.S.-G. All authors have read and agreed to the published version of the manuscript.

Funding: This research received no external funding.

Institutional Review Board Statement: This study was conducted in accordance with the tenets of the Helsinki Declaration and obtained Institutional Review Board approval. 
Informed Consent Statement: All patients included in this work were adequately informed, verbally and in writing, of the benefits, characteristics, and risks of the surgeries. All patients signed an informed consent form prior to the surgery and after the interview performed with the ophthalmologist.

Data Availability Statement: The data presented in this study are available on request from the corresponding author. The data are not publicly available due to be part of future research.

Conflicts of Interest: The authors declare no conflict of interest.

\section{References}

1. Wen, D.; McAlinden, C.; Flitcroft, I.; Tu, R.; Wang, Q.; Alió, J.; Marshall, J.; Huang, Y.; Song, B.; Hu, L.; et al. Postoperative Efficacy, Predictability, Safety, and Visual Quality of Laser Corneal Refractive Surgery: A Network Meta-analysis. Am. J. Ophthalmol. 2017, 178, 65-78. [CrossRef] [PubMed]

2. Chan, T.C.Y.; Ng, A.L.K.; Chan, K.K.W.; Cheng, G.P.M.; Wong, I.Y.H.; Jhanji, V. Combined application of prophylactic corneal cross-linking and laser in-situ keratomileusis-A review of literature. Acta Ophthalmol. 2017, 95, 660-664. [CrossRef]

3. Randleman, J.B.; Trattler, W.B.; Stulting, R.D. Validation of the Ectasia Risk Score System for Preoperative Laser in Situ Keratomileusis Screening. Am. J. Ophthalmol. 2008, 145, 813-818. [CrossRef] [PubMed]

4. Fernández, J.; Rodríguez-Vallejo, M.; Piñero, D.P. Tomographic and biomechanical index (TBI) for screening in laser refractive surgery. J. Refract. Surg. 2019, 35, 398. [CrossRef] [PubMed]

5. Ambrósio, R. Post-LASIK Ectasia: Twenty Years of a Conundrum. Semin. Ophthalmol. 2019, 34, 66-68. [CrossRef] [PubMed]

6. Lim, E.W.L.; Lim, L. Review of Laser Vision Correction (LASIK, PRK and SMILE) with Simultaneous Accelerated Corneal Crosslinking-Long-term Results. Curr. Eye Res. 2019, 44, 1171-1180. [CrossRef]

7. Spörl, E.; Huhle, M.; Kasper, M.; Seiler, T. Erhöhung der festigkeit der hornhaut durch vernetzung. Ophthalmologe 1997, 94, 902-906. [CrossRef] [PubMed]

8. Celik, H.U.; Alagöz, N.; Yildirim, Y.; Agca, A.; Marshall, J.; Demirok, A.; Yilmaz, O.F. Accelerated corneal crosslinking concurrent with laser in situ keratomileusis. J. Cataract Refract. Surg. 2012, 38, 1424-1431. [CrossRef]

9. Wollensak, G.; Hammer, C.M.; Spörl, E.; Klenke, J.; Skerl, K.; Zhang, Y.; Sel, S. Biomechanical efficacy of collagen crosslinking in porcine cornea using a femtosecond laser pocket. Cornea 2014, 33, 300-305. [CrossRef] [PubMed]

10. Kampik, D.; Ralla, B.; Keller, S.; Hirschberg, M.; Friedl, P.; Geerling, G. Influence of corneal collagen crosslinking with riboflavin and ultraviolet-a irradiation on excimer laser surgery. Investig. Ophthalmol. Vis. Sci. 2010, 51, 3929-3934. [CrossRef]

11. Chan, T.C.Y.; Yu, M.C.Y.; Ng, A.L.K.; Cheng, G.P.M.; Zhang, J.; Wang, Y.; Jhanji, V. Short-term variance of refractive outcomes after simultaneous LASIK and high-fluence cross-linking in high myopic correction. J. Refract. Surg. 2016, 32, 664-670. [CrossRef]

12. Kanellopoulos, A.J.; Asimellis, G. Combined laser in situ keratomileusis and prophylactic high-fluence corneal collagen crosslinking for high myopia: Two-year safety and efficacy. J. Cataract Refract. Surg. 2015, 41, 1426-1433. [CrossRef] [PubMed]

13. Kanellopoulos, J.; Asimellis, G.; Karabatsas, C. Comparison of prophylactic higher fluence corneal cross-linking to control, in myopic LASIK, one year results. Clin. Ophthalmol. 2014, 2373. [CrossRef]

14. Seiler, T.G.; Fischinger, I.; Koller, T.; Derhartunian, V.; Seiler, T. Superficial corneal crosslinking during laser in situ keratomileusis. J. Cataract Refract. Surg. 2015, 41, 2165-2170. [CrossRef] [PubMed]

15. Tomita, M.; Yoshida, Y.; Yamamoto, Y.; Mita, M.; Waring IV, G. In vivo confocal laser microscopy of morphologic changes after simultaneous LASIK and accelerated collagen crosslinking for myopia: One-year results. J. Cataract Refract. Surg. 2014, 40, 981-990. [CrossRef]

16. Kanellopoulos, A.J. Long-term safety and efficacy follow-up of prophylactic higher fluence collagen cross-linking in high myopic laser-assisted in situ keratomileusis. Clin. Ophthalmol. 2012, 6, 1125-1130. [CrossRef] [PubMed]

17. Tan, J.; Lytle, G.E.; Marshall, J. Consecutive laser in situ keratomileusis and accelerated corneal crosslinking in highly myopic patients: Preliminary results. Eur. J. Ophthalmol. 2014, 25, 101-107. [CrossRef] [PubMed]

18. Kohnen, T.; Lwowski, C.; Hemkeppler, E.; De'Lorenzo, N.; Petermann, K.; Forster, R.; Herzog, M.; Böhm, M. Comparison of Femto-LASIK with Combined Accelerated Cross-linking to Femto-LASIK in High Myopic Eyes: A Prospective Randomized Trial. Am. J. Ophthalmol. 2020, 211, 42-55. [CrossRef] [PubMed]

19. Wu, Y.; Tian, L.; Wang, L.Q.; Huang, Y.F. Efficacy and Safety of LASIK Combined with Accelerated Corneal Collagen Cross-Linking for Myopia: Six-Month Study. BioMed Res. Int. 2016, 2016. [CrossRef] [PubMed]

20. Bradley Randleman, J.; Santhiago, M.R.; Kymionis, G.D.; Hafezi, F. Corneal cross-linking (CXL): Standardizing terminology and protocol nomenclature. J. Refract. Surg. 2017, 33, 727-729. [CrossRef]

21. Ng, A.L.K.; Kwok, P.S.K.; Wu, R.T.K.; Jhanji, V.; Woo, V.C.P.; Chan, T.C.Y. Comparison of the Demarcation Line on ASOCT after Simultaneous LASIK and Different Protocols of Accelerated Collagen Crosslinking: A Bilateral Eye Randomized Study. Cornea 2017, 36, 74-77. [CrossRef] [PubMed]

22. Taneri, S.; Kiessler, S.; Rost, A.; Dick, H.B. Corneal ectasia after LASIK combined with prophylactic corneal cross-linking. J. Refract. Surg. 2017, 33, 50-52. [CrossRef] [PubMed]

23. Kanellopoulos, A.J.; Asimellis, G.; Salvador-Culla, B.; Chodosh, J.; Ciolino, J.B. High-irradiance CXL combined with myopic LASIK: Flap and residual stroma biomechanical properties studied ex-vivo. Br. J. Ophthalmol. 2015, 99, 870-874. [CrossRef] [PubMed] 